\title{
LA DANZA DE LOS ESQUELETOS: LA ENSEÑANZA DE LA BIOARQUEOLOGÍA A LOS NIÑOS Y AL PÚBLICO EN GENERAL
}

\section{DANCE OF THE SKELETONS: TEACHING BIOARCHAEOLOGY TO CHILDREN AND OVERALL PUBLIC}

\author{
Sandra Assis ${ }^{1,2}$ y Ana Luísa Santos ${ }^{2}$
}

\begin{abstract}
RESUMEN
Los científicos tienen un importante rol en el desarrollo de interfaces entre el conocimiento científico producido y las escuelas y/o el público en general. En Portugal, sin embargo, la transferencia de conocimiento entre las universidades y el público fue una actividad desvalorizada durante décadas. Con el objetivo de contrarrestar esta tendencia, una organización científica sin fines de lucro llamada Grupo de Estudio en Evolución Humana, con lugar de trabajo en el Departamento de Ciencias de la Vida de la Universidad de Coimbra, ha desarrollado desde 2005 un conjunto de actividades/talleres pedagógicos y experimentales en Portugal y en el extranjero en el campo de antropología biológica, bioarqueología y evolución humana. Entre ellas, el taller "La danza de los esqueletos" tiene el objetivo de introducir el rol del esqueleto en el balance fisiológico del cuerpo humano, así como aclarar la importancia de recuperar restos de esqueletos humanos a partir de contextos arqueológicos para reconstruir la historia y la evolución de poblaciones pasadas. El componente práctico de éste consiste en un juego sobre un tablero humano gigante, compuesto de preguntas acerca de la biología y anatomía de un esqueleto humano, que finaliza en la simulación de una excavación en una caja de arena. Nuestra experiencia en Portugal nos permite concluir que este tipo de acercamientos es bien aceptado por los estudiantes (de escuela primaria y secundaria) y maestros, constituyendo un complemento óptimo de la enseñanza formal. Estos talleres pueden ser fácilmente adaptados; de acuerdo con esto, invitamos a los investigadores a desarrollar estas actividades en sus países.
\end{abstract}

Palabras clave: anatomía humana, la educación no formal; antropología biológica; arqueología; servicio educativo

\section{ABSTRACT}

Scientists play an important role in developing interfaces between the scientific knowledge produced and the schools and/or general public. In spite of its importance, the transference of knowledge between universities and the public has been neglected in Portugal during decades. Aiming to suppress this tendency, a nonprofit scientific organization called Group of Studies in Human Evolution (GEEvH) housed at the Department of Life Sciences of the University of Coimbra has developed, since 2005, a set of pedagogical and experimental activities/workshop in Portugal and abroad

Tipología: Articulo de reflexión

Fecha de recepción: 13/02/2014

Fecha de aceptación: 11/07/2014

Como citar éste artículo: Assis, S., \& Santos, A. (2014). La danza de los esqueletos: la enseñanza de la bioarqueología a los niños y al público en general. Jangwa Pana, 13, 179 - 185

1. Grupo de Estudos em Evolução Humana, GEEvH. University of Coimbra, Portugal. Correo: alsantos@antrop.uc.pt

2. CIAS and Department of Life Sciences, University of Coimbra, Portugal. Correo: greevh@gmail.com 


\section{ABSTRACT}

on the field of biological anthropology/bioarcheology and human evolution. Of the activities developed, the workshop "Dance of the skeletons" aims to introduce the role of the skeleton in the physiological balance of the human body, as well as to clarify the importance of recovering human skeletal remains from archaeological contexts to reconstruct past populations' history and evolution. The practical component of it consists in a giant human roll-and-move game composed of questions about the biology and anatomy of the human skeleton that finishes in the simulation of an excavation in a sandbox. Our experience in Portugal led us to conclude that this type of approach is well accepted by the students (from elementary to high school) and teachers, constituting an optimal complement to the formal teaching. These ateliers/workshops can be easily adapted; accordingly we would like to invite researchers to develop these activities in their countries.

Keywords: human anatomy, non-formal education; biological anthropology; archeology; educational service

\section{INTRODUCCIÓN}

$\mathrm{L}$ a ciencia, y el conocimiento producido, están en constante transformación. Es innegable el papel que la escuela tiene en la enseñanza y la promoción de la ciencia. Sin embargo, dada la rapidez con que se desarrollan muchos descubrimientos, a veces resulta difícil para los docentes hacer una actualización de los nuevos conocimientos. En este proceso, los científicos juegan un papel muy importante en la creación de interfaces que permiten la transferencia de conocimiento entre las universidades, los centros de investigación, las escuelas y el público en general. El compromiso que los investigadores tienen con la sociedad se basa no sólo en la producción científica, sino también en la creación de canales de comunicación que faciliten la transmisión de los conocimientos adquiridos (Honeyman 1998). Además de la educación formal, los investigadores deberían participar activamente en la creación de mecanismos informales y no formales de la divulgación de la ciencia en colaboración con los medios de comunicación, centros de ciencia y museos, programas de educación de la comunidad, entre otros (Honeyman 1998; Bloom 2006). A pesar de la reconocida importancia de la transferencia de saberes para la sustentabilidad científica, Portugal se caracterizó por el prolongado distanciamiento entre el conocimiento producido en las universidades y el público en general. Esta situación comenzó a cambiar progresivamente desde 1996 con la creación de Ciencia VivaAgencia Nacional para la Cultura Científica y Tecnológica (http://www.cienciaviva.pt/home/). Esta plataforma gubernamental aboga por la promoción de la cultura científica y la tecnología en la sociedad portuguesa, en este caso junto con la población escolar. Con el objetivo de descentralizar y difundir el conocimiento científico, fueron creados 20 centros interactivos "Ciencia Viva" en diferentes regiones de Portugal, subordinados a diferentes temáticas (por ejemplo astronomía, física, ecología y ambiente, recursos naturales y robótica, entre otros). Paralelamente, la plataforma Ciencia Viva se dedicó a fomentar el desarrollo de proyectos escolares y/o provenientes de otras áreas de la sociedad que promocionan la difusión del conocimiento científico. Dada la inexistencia de actividades de divulgación en el dominio de la evolución humana y de la bioarqueología, el Grupo de Estudios en Evolución Humana $(\mathrm{GEEvH})$ elaboró un proyecto pionero intitulado "Jugar con el Gran Árbol de la Evolución" (CV/ PVI 1845), aprobado en 2006 en el ámbito de la IV edición del concurso Ciencia Viva.

En este artículo se pretende: (1) realizar una breve introducción a los objetivos y actividades desarrollados por el Grupo de Estudios en Evolución Humana y (2) presentar el taller La danza de los esqueletos, especialmente planificado para la divulgación de la bioarqueología, haciendo refe- 
rencia a los objetivos, estructura de las actividades y niveles de aceptación de la comunidad.

\section{El GEEvH - Grupo de Estudios en Evolución Humana}

El GEEvH [http://geevh.jimdo.com/] es una asociación sin fines de lucro fundada en 2005 por los alumnos de la antigua Maestría en Evolución Humana (Edición 2004-2006) del anterior Departamento de Antropología, actualmente Departamento de Ciencias de la Vida de la Universidad de Coimbra, que tiene como principal misión la promoción, el desarrollo y la difusión de la investigación en el campo de la evolución humana.

Para lograr los objetivos propuestos, el GEEvH desarrolló, en el ámbito del concurso nacional Ciencia Viva VI, el proyecto "Jugar con el Gran Árbol de la Evolución” (CV/PVI 1845), compuesto por una serie de talleres pedagógicos que consideraron tres grandes ramas de la ciencia cuyo papel es fundamental para el estudio de la evolución humana: la biología, la antropología biológica en sus diferentes subdisciplinas (por ejemplo la paleoantropología, la bioarqueología y la primatología) y la arqueología (Tabla 1). Cada taller pedagógico comprende dos partes. La primera incluye un componente de presentación multimedia y de interactividad tecnológica, que pretende transmitir a los niños un conjunto de conocimientos útiles para llevar a cabo los experimentos y las actividades lúdicas que ocuparán la segunda parte de las actividades. La aceptación gubernamental del proyecto ha posibilitado la adquisición del material didáctico necesario para su implementación. De todas formas, resulta pertinente aclarar que todos los talleres pedagógicos se realizaron mediante un régimen de voluntariado. Las actividades fueron implementadas por los miembros y socios colaboradores del GEEvH en museos (antiguo Museo Antropológico de la Universidad de Coimbra - Coimbra, región centro de Portugal; Museo D. Diogo de Sousa - Braga, región norte de Portugal), organismos municipales (Cámara Municipal de Leiria, centro de Portugal, en el ámbito de la exposición Habitantes y hábitats; Cámara Municipal de Vila Nova de Foz Côa/Parque Arqueológico del Valle de Côa, norte de Portugal, en el ámbito de las actividades de vacaciones escolares) y en grupos escolares de todo el país. También se realizaron exhibiciones en ferias de ciencias regionales y nacionales. Durante el desarrollo de las actividades se procuró siempre establecer una adecuación entre los contenidos teóricos/lenguaje utilizado y la audiencia.

\section{La danza de los esqueletos: acercamiento informal a la enseñanza de la bioarqueología}

Entre las actividades llevadas a cabo por el GEEvH se destaca el taller dedicado a la bioarqueología intitulado "La danza de los esqueletos". Este taller tuvo como objetivo mostrar la importancia que el esqueleto tiene en vida, en su participación en numerosos procesos que garantizan el equilibrio biológico de nuestro cuerpo, y en paralelo mostrar cuán importante es su recuperación y estudio en su contexto arqueológico, ya que nos ofrece una perspectiva diferente acerca de las poblaciones del pasado.

El taller tuvo un componente teórico que ofreció algunos conceptos básicos de osteología humana con el fin de responder a preguntas como (Figura 1):

- ¿Qué es el sistema esqueletal?

- ¿Cómo están constituidos los huesos?

- ¿Cómo ocurre el desarrollo del esqueleto desde el momento de la fecundación hasta la fase adulta?

- ¿Cuántos huesos tienen los niños y los adultos?

- ¿Cuáles son las funciones del esqueleto?

- ¿Cuáles son y para qué sirven las articulaciones del esqueleto? 
Tabla 1. Presentación y descripción de los talleres llevados a cabo por el GEEvH

\begin{tabular}{|c|c|c|c|}
\hline $\begin{array}{c}\text { Rama } \\
\text { científica }\end{array}$ & Subdisciplinas & Título del taller & Tipo de actividad realizada \\
\hline Biología & & $\begin{array}{l}\text { Evolución: en el } \\
\text { inicio éramos } \\
\text { un... }\end{array}$ & \multirow{5}{*}{$\begin{array}{l}\text { - Presentación teórica corta } \\
\text { para introducir los temas y } \\
\text { conceptos principales. } \\
\text { - Actividades prácticas e } \\
\text { interactivas experimentos } \\
\text { "hands-on" y/o juegos } \\
\text { informales para aplicar los } \\
\text { conceptos aprendidos. }\end{array}$} \\
\hline \multirow{3}{*}{$\begin{array}{l}\text { Antropología } \\
\text { biológica }\end{array}$} & Paleoantropología & $\begin{array}{l}\text { Fósiles: los } \\
\text { abuelos de } \\
\text { todos nosotros }\end{array}$ & \\
\hline & Primatología & Monerías & \\
\hline & Bioarqueología & $\begin{array}{l}\text { La danza de los } \\
\text { esqueletos }\end{array}$ & \\
\hline Arqueología & & $\begin{array}{l}\text { Dibujos y } \\
\text { garabatos: } \\
\text { el arte en la } \\
\text { Prehistoria }\end{array}$ & \\
\hline
\end{tabular}

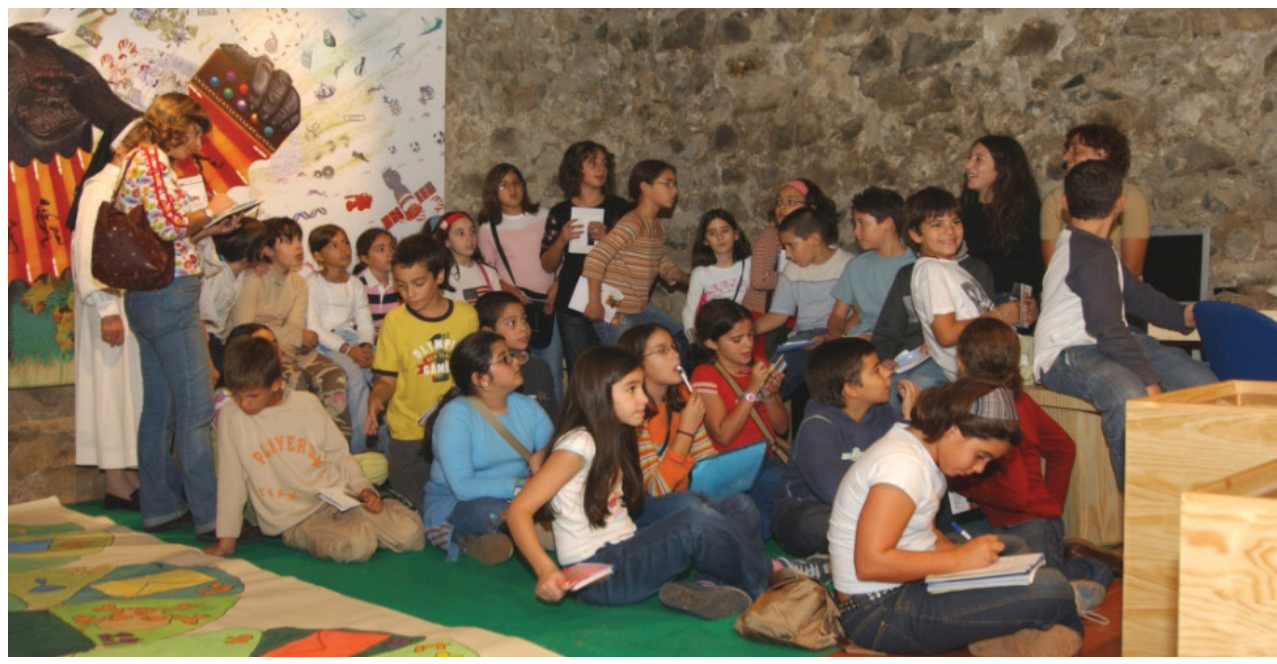

Figura 1. Exposición de los contenidos teóricos del taller pedagógico "La danza de los esqueletos" (@ Cámara Municipal de Leiria)

Paralelamente, tuvo como objetivo:

Introducir al alumno en el trabajo de campo y de laboratorio del bioarqueólogo, mostrando el tipo de información que se puede "leer" a partir de los vestigios osteológicos humanos antiguos, tales como: el sexo, la edad de las personas, su ascendencia y algunas patologías, entre otros.

Aclarar la importancia de recuperar los vestigios osteológicos humanos provenientes de contextos arqueológicos con el fin de reconstruir la historia y la evolución de los grupos humanos extintos. 
El componente práctico consistió en un juego pedagógico denominado " $\mathrm{La}$ danza de los huesos", que tuvo como objetivo crear un dispositivo dinámico y divertido que motiva la aplicación de los conocimientos adquiridos durante la unidad teórica. El juego mencionado es un "roll-and-move" humano de gran tamaño (Figura 2), que consiste en un circuito de preguntas y desafíos teóricos que culmina en la simulación de una excavación arqueológica/antropológica en una caja de arena (Figura 3).

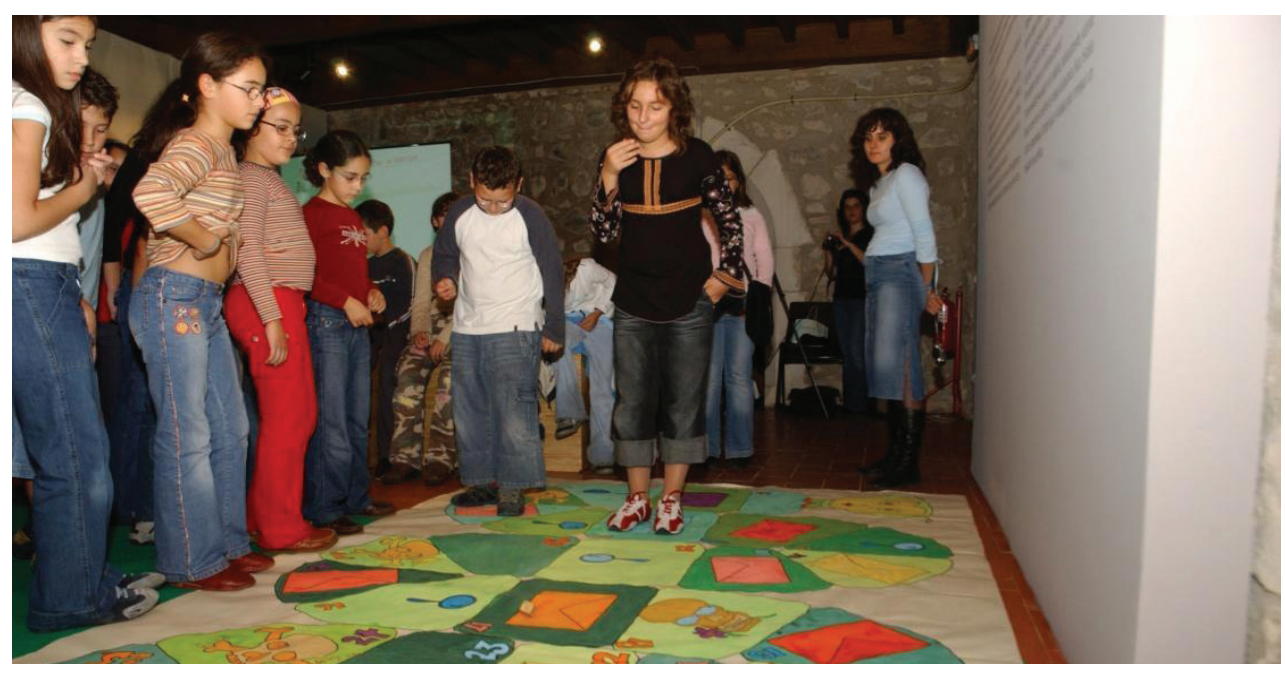

Figura 2. Juego "La danza de los huesos" en acción (@ Cámara Municipal de Leiria)

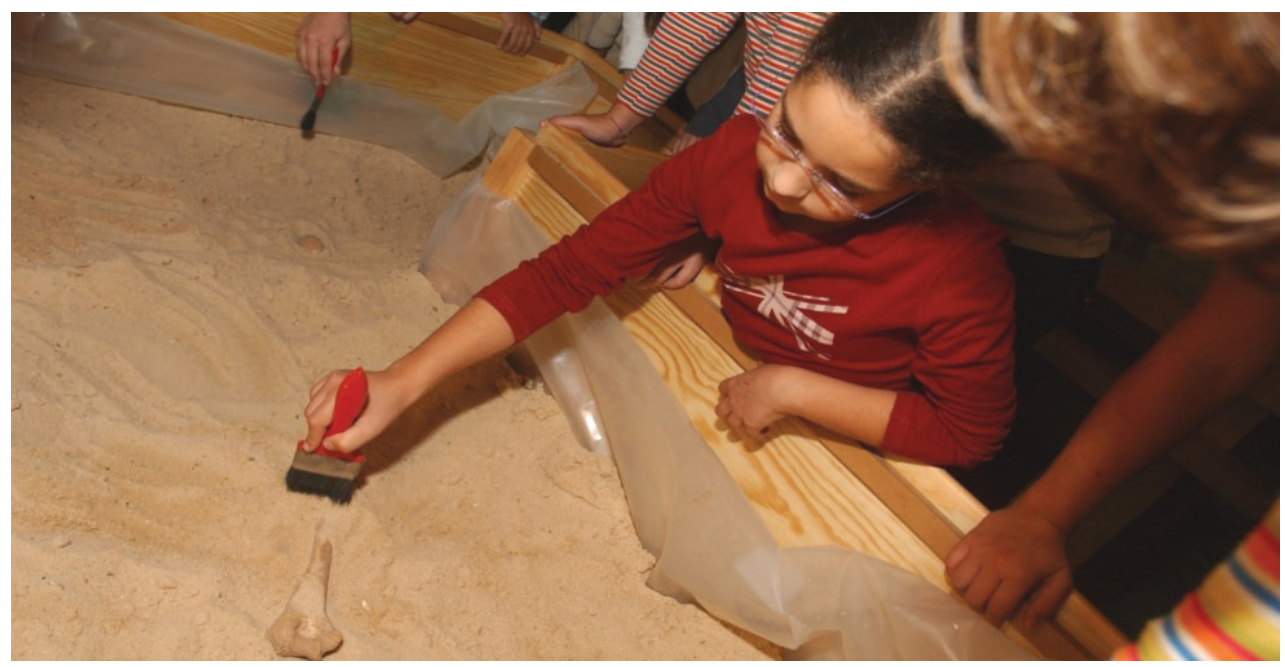

Figura 3. Simulación de una excavación en caja de arena (@ Cámara Municipal de Leiria)

\section{El juego “Danza de los huesos”: reglas para la diversión!}

Para comenzar el juego son necesarios dos equipos formados al comienzo del taller. Cada equipo es responsable de la selección de cuatro representan- 
tes: uno de ellos debe retirar las consignas durante la exposición teórica, el segundo debe lanzar el dado que determinará el número de casillas a avanzar, el tercero es el "peatón-humano", cuya función es la de circular entre las distintas casillas y el cuarto tiene la función de responder las preguntas con la ayuda de otros miembros del equipo. Esta estrategia de juego tiene como objetivo promover la división de tareas y la cooperación dentro de los equipos. Las reglas del juego son simples. Cada equipo tira los dados por primera vez. El equipo que obtiene el valor más alto es el que comienza el juego. El equipo ganador realiza un segundo lanzamiento, que indicará la cantidad de casillas que debe avanzar. Las casillas del juego no son todas iguales. Algunas tienen preguntas o premios, otras pueden penalizar a los equipos. Por ejemplo, una de las casillas está representada por un cráneo pirata. Si uno de los equipos llega a esta casilla, será penalizado y caerá de nuevo en la anterior. Si se llega a la casilla que tiene un cráneo y un trébol, el equipo se beneficiará avanzando a la siguiente casilla. En caso de llegar a la casilla con flores, automáticamente se obtiene la victoria. Si se llega a la casilla que tiene un dibujo de una bomba, el equipo debe volver al punto de partida. Siempre que un equipo responde correctamente a una de las preguntas, avanza a la casilla siguiente, y si la respuesta es incorrecta, permanece en la misma casilla.

\section{Comentarios finales}

Durante más de cinco años de actividad, alrededor de 1.678 estudiantes y un sinnúmero de otros participantes se han beneficiado de este modelo de difusión del conocimiento en Portugal (Assis \& Carvalho, 2012) y en Guinea Conacry. Se trató de un proyecto pionero de divulgación de la ciencia que se focalizó, por primera vez, en la temática de la evolución humana y de la bioarqueología, áreas frecuentemente ausentes $\mathrm{y} / \mathrm{o}$ desvalorizadas en los manuales escolares portugueses (Assis \& Carvalho, 2012). El hecho de que las actividades se desarrollaran dentro de un ámbito interactivo y no formal condujo a la participación dinámica de todos los intervinientes: alumnos, profesores y moderadores. En el desarrollo de todo el proceso se fueron realizando adecuaciones de contenidos, no solo en relación con los nuevos descubrimientos científicos sino también considerando la franja etaria y las necesidades curriculares de los alumnos. De hecho, para muchos grupos escolares los talleres pedagógicos funcionaron como un complemento de la currícula escolar. En el caso específico del taller La danza de los esqueletos, además de fortalecer la información sobre la biología del esqueleto, permitió introducir a los participantes en una nueva realidad de investigación poco conocida en Portugal, la bioarqueología. En términos generales, los obstáculos más importantes en la ejecución del proyecto tuvieron que ver con el carácter voluntario y con las limitaciones gubernamentales impuestas a la realización y/o la participación de las escuelas en estas actividades extraccurriculares. Además, otra de las dificultades que se presentaron en el lugar de realización de los talleres se produjo en el ejercicio de las actividades para el público con necesidades especiales. No obstante, es posible concluir que este tipo de abordaje no formal de enseñanza de la ciencia fue muy bien aceptado por los alumnos (niveles elemental y secundario), profesores y público en general, constituyendo un complemento óptimo para la enseñanza formal. Estos talleres tienen la ventaja de adaptarse fácilmente de acuerdo con la realidad y las necesidades educativas de diversos públicos y/o países. En consecuencia, nos gustaría invitar a todos los investigadores interesados a que lleven adelante este tipo de actividades, porque: la ciencia no es gris, sino que muy por el contrario, es, y deberá ser, siempre divertida!

\section{AGRADECIMIENTOS}

A todos los alumnos, profesores y demás público que aceptó formar parte de esta danza. A Claudia Aranda, Leandro Luna y Jorge Suby. A todos los organismos públicos que colaboraron en la divul- 
gación de esta iniciativa: Programa Ciência Viva, Cámara Municipal de Leiria, CIAS - Centro de Investigação em Antropologia e Saúde y FCT.

\section{REFERENCIAS BIBLIOGRÁFICAS}

Assis, S., \& Carvalho, S. (2012). Evolutionism and the Teaching of Science: How Portugal Has Been "Playing with the Big Tree of Evolution". Evolution Education and Outreach 5: 445-452.
Honeyman, B. (1998). Non-formal and formal learning interactions: new directions for scientific and technological literacy. Connect-UNESCO. The International Science Technology and Environmental Education Newsletter. 23 (1):1-16.

Bloom, J. (2006). Creating a classroom community of young scientists. New York: Routledge. 